Article

\title{
Bioactive Compounds in Kimchi Improve the Cognitive and Memory Functions Impaired by Amyloid Beta
}

\author{
Minji Woo ${ }^{1}\left(\mathbb{D}\right.$, Mi Jeong $\mathrm{Kim}^{2}$ and Yeong Ok Song ${ }^{1, *(\mathbb{D})}$ \\ 1 Department of Food Science and Nutrition and Kimchi Research Institute, Pusan National University, \\ Busan 46241, Korea; woo07140@pusan.ac.kr \\ 2 Department of Food and Nutrition, Silla University, Busan 46958, Korea; mjkim@silla.ac.kr \\ * Correspondence: yosong@pusan.ac.kr; Tel.: +82-51-510-2847; Fax: +82-51-583-3648
}

Received: 18 September 2018; Accepted: 18 October 2018; Published: 20 October 2018

check for updates

\begin{abstract}
This study investigated the abilities of kimchi and its bioactive compounds to ameliorate amyloid beta $(\mathrm{A} \beta)$-induced memory and cognitive impairments. Mice were given a single intracerebroventricular injection of $A \beta_{25-35}$, followed by a daily oral administration of capsaicin (10 mg.kg-bw $\left.{ }^{-1}\right)$, 3-(4'-hydroxyl-3', $5^{\prime}$-dimethoxyphenyl)propionic acid (50 mg/kg bw), quercetin (50 mg/kg bw), ascorbic acid (50 mg/kg bw), or kimchi methanol extract (KME; $200 \mathrm{mg} / \mathrm{kg}$ bw) for 2 weeks ( $n=7$ per group). Carboxymethylcellulose was used as a vehicle for the normal and control groups. Behavioral task tests showed that the learning and memory abilities were significantly waned by the injected $A \beta_{25-35}$, but these cognitive deficits were recovered by the administrated KME and kimchi bioactive compounds $(p<0.05)$. The reactive oxygen species, peroxynitrite, and thiobarbituric acid reactive substances levels were lower, and the glutathione level was higher, in the KME and bioactive compound groups than in the control group $(p<0.05)$. In the KME and bioactive compound groups, the protein expression levels of antioxidant enzymes (nuclear factor (erythroid-derived 2)-like 2-regulated superoxide dismutase-1 and glutathione peroxidase) were increased, whereas those of inflammation-related enzymes (nuclear factor-kappaB -regulated inducible nitric oxide synthase and cyclooxygenase-2) were decreased $(p<0.05)$. Thus, the antioxidative and anti-inflammatory properties of bioactive compounds-rich kimchi might help to attenuate the symptoms of Alzheimer's disease.
\end{abstract}

Keywords: Alzheimer's disease; amyloid beta; behavioral test; cognitive deficits; kimchi; oxidative stress; inflammation

\section{Introduction}

Cognitive deficits with memory losses and lack of recognition are typical signs of Alzheimer's disease (AD), which subsequently brings about a behavioral change in afflicted individuals. It is well established that accumulation of the amyloid beta $(A \beta)$ peptide in the brain is an immediate cause of synaptic dysfunction and neuronal cell death in AD. Nonetheless, oxidative stress and inflammation are recognized as important causative factors as well [1]. A $\beta$ peptides play an important role in the neuroinflammatory responses mediated through reactive oxygen species (ROS) generation. In the pathogenesis of $\mathrm{AD}$, the amyloid-cascade hypothesis and the oxidative-stress hypothesis are considered as one concept by many authors [1].

Behavioral task tests are among the tools used to evaluate cognitive deficits in the mouse model of $\mathrm{AD}[2,3]$, with the T-maze, novel object recognition, and Morris water maze tests being the most widely applied. Specifically, the novel object recognition test [4] and the T-maze test [5] are designed to evaluate cognitive ability through observation of the subject's exploratory behaviors toward a new 
object or route, respectively. The Morris water maze task test is designed to evaluate learning and memory levels, using a swimming pool with a hidden platform [6].

Natural products rich in polyphenols with multiple functions, in particular antioxidative activity, might potentially hinder neurodegeneration and thereby improve memory and cognitive functions through the alleviation of $A \beta$-induced neurotoxicity [7]. Numerous studies have shown the preventive or curable functions of natural products and bioactive compounds against $A D$, such as the anti-amyloidogenic activities of resveratrol, gingerols, curcumin, and gallate in models of the disease [8]. Quercetin has also been reported for its neuroprotective effects mediated through the attenuation of oxidative stress [9] and inflammation [10] and reduction of proteins related to AD [10].

Kimchi, a Korean traditional fermented vegetable, contains numerous bioactive compounds because it is prepared with cabbage, red pepper, garlic, ginger, green onion, and fermented fish sauce. Consequently, kimchi has demonstrated various health-beneficial effects, including the prevention of oxidative stress [11], inflammation [12], and cancer [13], and improvement of cognitive abilities [3]. These effects of kimchi might be attributed to the antioxidative activity of its component bioactive compounds [14]. In our previous study, several bioactive compounds were identified from the kimchi methanol extract (KME); namely, ascorbic acid, capsaicin, 3-(4'-hydroxyl-3',5'-dimethoxyphenyl)propionic acid (HDMPPA), and quercetin, at 280, 270, 40, and $20 \mathrm{mg} / \mathrm{kg}-\mathrm{KME}$, respectively [15]. Capsaicin, ascorbic acid, and HDMPPA are monophenol compounds, whereas quercetin is a polyphenol compound. In this study, the neuroprotective effects of KME and its bioactive compounds were investigated in a mouse model of $A \beta_{25-35}$-induced AD. Behavioral task tests were performed in addition to biochemical evaluation of the protein expression of antioxidants and inflammatory cytokines in the treated animals.

\section{Materials and Methods}

\subsection{Kimchi Bioactive Compounds and Kimchi Methanol Extract}

The individual kimchi bioactive compounds that were previously identified by our team to be present in KME [15] were purchased from Sigma-Aldrich (St. Louis, MO, USA); namely, HDMPPA (CDS011403), quercetin (Q4951, purity $\geq 95 \%$ ), ascorbic acid (A5960, purity $\geq 99 \%$ ), and capsaicin (M2028, purity $\geq 95 \%$ ). The KME was prepared for the present study as described below.

Kimchi was first made by mixing together the following ingredients: Brined cabbage $(84.9 \%)$, red pepper powder $(2.6 \%)$, garlic $(2.5 \%)$, green onion $(2.3 \%)$, ginger $(0.5 \%)$, fermented fish sauce $(3.0 \%)$, sugar $(0.5 \%)$, and glutinous rice paste $(3.7 \%)$. The mixture was fermented at $10{ }^{\circ} \mathrm{C}$ for a day and stored at $0{ }^{\circ} \mathrm{C}$ in a kimchi refrigerator for 14 days (R-K182PM; LG, Seoul, Korea) until the pH reached $4.3 \pm 0.1$. The resultant kimchi was then freeze-dried (SFDSM06; Samwon Co., Busan, Korea). The KME was prepared by three repeated extractions of the freeze-dried kimchi with $70 \%$ methanol, for $24 \mathrm{~h}$ at room temperature each time. All extracts were combined together, concentrated using a rotary evaporator (R-200; Buchi, Flawil, Switzerland), and then freeze-dried.

\subsection{Animals and Intracerebroventricular Injection of $A \beta_{25-35}$}

ICR mice (male, 5 weeks old) were purchased from Orient, Inc. (Seongnam, Korea). The mice were kept under a controlled temperature $\left(23 \pm 1{ }^{\circ} \mathrm{C}\right)$ and humidity $(50 \pm 5 \%)$ with a $12 \mathrm{~h}$ light-dark cycle, and fed chow diet and water ad libitum. After 1 week of acclimatization, the animals were assigned to seven groups ( $n=7$ per group) on the basis of body weight (bw). A $\beta_{25-35}$ (Sigma-Aldrich) dissolved in phosphate-buffered saline (PBS) was incubated at $37^{\circ} \mathrm{C}$ for 3 days. On day 0 , the mice in the experimental groups were given an intracerebroventricular injection of aggregated $A \beta_{25-35}$, except for the animals in the normal (NOR) group, which were injected with PBS instead. In brief, a $5 \mu \mathrm{L} \mathrm{A} \beta_{25-35}$ solution ( $5 \mathrm{nmol} /$ mouse) or PBS was injected into the bregma ( $2.2 \mathrm{~mm} \mathrm{depth}$ ) of each mouse, using a $10 \mu \mathrm{L}$ Hamilton microsyringe equipped with a 26-gauge needle [1,16]. Then, from day 5 onward, capsaicin (at $10 \mathrm{mg} / \mathrm{kg}$ bw/day), HDMPPA, quercetin, ascorbic acid (at $50 \mathrm{mg} / \mathrm{kg}$ 
bw/day each), or KME (at $200 \mathrm{mg} / \mathrm{kg}$ bw/day), each dissolved in carboxymethylcellulose, was orally administered to the mice for 2 weeks. The NOR group and an $\mathrm{A} \beta_{25-35}$-injected control (CON) group received the carboxymethylcellulose vehicle only. The treatment dosage was determined according to previous studies $[10,17,18]$, where the dosage of capsaicin was further adjusted since the compound demonstrated toxicity at a high dose [19]. The dosage of KME was based on that used in previous studies at which it exhibited positive effects in the mouse model [2,15]. From day 14, three different behavioral tests were performed for 8 days according to previous studies [1,2,20]; that is, 4 consecutive days for the Morris water maze test, 2 days for the novel object recognition test, and another 2 days for the T-maze test. On day 19, all mice were fasted for $12 \mathrm{~h}$ and then sacrificed after $\mathrm{CO}_{2}$ anesthetization. The experimental schedule for the animal study is shown in Figure 1. The organs were perfused with ice-cold PBS and excised. The animal protocols were reviewed and approved by the Institutional Animal Care and Use Committee of Pusan National University (Approval No. PNU-2016-1385).

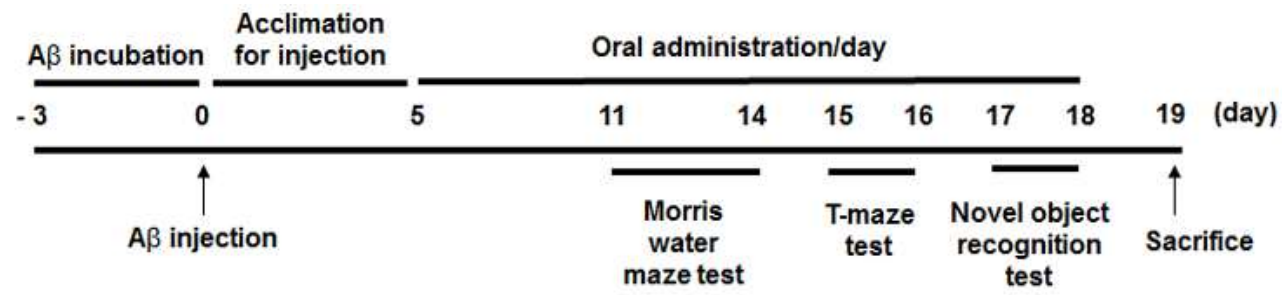

Figure 1. Experimental schedule for the mouse injections with $A \beta_{25-35}$.

\subsection{Morris Water Maze Test}

Memory function was examined with a slightly modified Morris water maze test [6]. The circular pool used for the test was $100 \mathrm{~cm}$ in diameter and $35 \mathrm{~cm}$ in height. During the experiment, the water temperature was maintained at $22 \pm 1{ }^{\circ} \mathrm{C}$. For the behavioral test, the water pool was divided into quadrants. A platform $(8 \mathrm{~cm}$ in diameter) was placed $1 \mathrm{~cm}$ underneath the water at one designated quadrant, and visual signs were marked on the other quadrants for spatial navigation. White paint (water-soluble, nontoxic) was added to make the water opaque. A total of 12 trials were conducted, three times a day. The time taken to search for the hidden platform was recorded up to $60 \mathrm{~s}$. After each performance, the mouse was allowed to rest on the platform for $15 \mathrm{~s}$ to remember the comfort of the environment regardless of mission completion. On day 4, the 10th trial was performed as usual, using a hidden platform. However, the 11th trial was carried out without the platform. The times taken for staying to find the target quadrant were measured, respectively. For the final (12th) trial, the performance test was carried out in clear water to investigate whether the latency time would be different under the more visible environment.

\subsection{Novel Object Recognition Test}

Recognition of a new object was tested using a black-colored cage $(40 \times 40 \times 40 \mathrm{~cm})$ [4]. For the 1st trial, two identical objects $\left(\mathrm{A}, \mathrm{A}^{\prime}\right)$ were placed in the box, and the frequency of touching each object was recorded for $10 \mathrm{~min}$, separately. In the 2 nd test conducted after $24 \mathrm{~h}$, one of the old objects used in the 1st trial was replaced with a new object (A, B). Novel object recognition was calculated as the ratio of the number of times touching the familiar object or new object to the sum of the touching frequencies.

\subsection{T-maze Test}

Memory function was examined using a T-maze test [5]. The T-shaped black box had two routes (the left and right arms, respectively) and a starting position at the front pouch. The left-hand-side door was designed to be open all the time, and the right-hand-side door was closed in the 1st test but opened in the 2nd test. The frequencies of touching the gate for exploring each route were recorded for $10 \mathrm{~min}$. The time lap between the two trials was $24 \mathrm{~h}$. 
2.6. Reactive oxygen species, Peroxynitrite, Thiobarbituric Acid Reactive Substances, and Glutathione Levels in the Brain

Brain homogenates were prepared in PBS $(1: 9, w / v)$. The thiobarbituric acid reactive substances (TBARS) levels in the homogenates were determined using a malondialdehyde standard curve, and the glutathione (GSH) levels were measured using a GSH standard curve [15].

A post-mitochondrial fraction was obtained by centrifugation of the brain homogenate, and the ROS and peroxynitrite levels were determined in this fraction using $2^{\prime}, 7^{\prime}$-dichlorofluorescein diacetate [21] and dihydrorhodamine 123 buffer [22], respectively. Changes in the fluorescence of the reaction samples were measured at 480 and $535 \mathrm{~nm}$ for $30 \mathrm{~min}$ with a fluorescence plate reader (FLUOstar OPTIMA; BMG LABTECH, Ortenberg, Germany).

\subsection{Western Blot Analysis}

The activities of antioxidative and anti-inflammatory enzymes in the brain tissue were determined by western blot assay as described previously [23]. Enzyme protein expression was visualized by enhanced chemiluminescence, measured with a CAS-400 system (Core Bio, Seoul, Korea), and calculated using ImageJ software (National Institutes of Health, Bethesda, MD, USA). The amount of each target protein was normalized to that of alpha-tubulin according to band density. The primary antibodies to nuclear factor (erythroid-derived 2)-like 2 (Nrf2, sc-13032), superoxide dismutase-1 (SOD1, sc-11407), glutathione peroxidase (GPx, sc-133160), nuclear factor-kappaB (NF-kB, sc-109), inducible nitric oxide synthase (iNOS, sc-651), and cyclooxygenase-2 (COX-2, sc-1747) were purchased from Santa Cruz Biotechnology (Santa Cruz, CA, USA). The donkey polyclonal secondary antibody to rabbit IgG (ab6802), goat polyclonal secondary antibody to mouse IgG (ab6789), and donkey polyclonal secondary antibody to goat IgG (ab6885) were purchased from Abcam Inc. (Cambridge, UK).

\subsection{Statistical Analysis}

Values are presented as the mean \pm standard deviation. Significant differences between the CON group and each experimental group were assessed by one-way analysis of variance followed by Dunnett's multiple-comparison test, using SPSS version 22.0 software (SPSS Inc., Chicago, IL, USA). Student's $t$-test was also applied to the data from the object recognition task test and T-maze task test. A value of $p<0.05$ was considered statistically significant.

\section{Results}

\subsection{Morris Water Maze Test}

Body weight changes among the $\mathrm{A} \beta_{25-35}$-injected groups were not significantly different. The time taken by the experimental groups to reach the hidden platform decreased considerably with increased training time, compared with that taken by the CON group (Figure 2A). The latency time to the platform was the longest for the CON group, followed by the capsaicin, ascorbic acid, HDMPPA, quercetin, KME, and NOR groups, in order. The occupancy time in the target quadrant where the platform was originally placed was longer for the experimental groups than for the CON group, but significant difference was found only with the quercetin and KME groups ( $p<0.05$, Figure 2B). However, there were no significant differences in the latency to reach the exposed platform among the experimental groups indicating that the improvement of memory function is not associated with the visual ability (Figure 2C). 

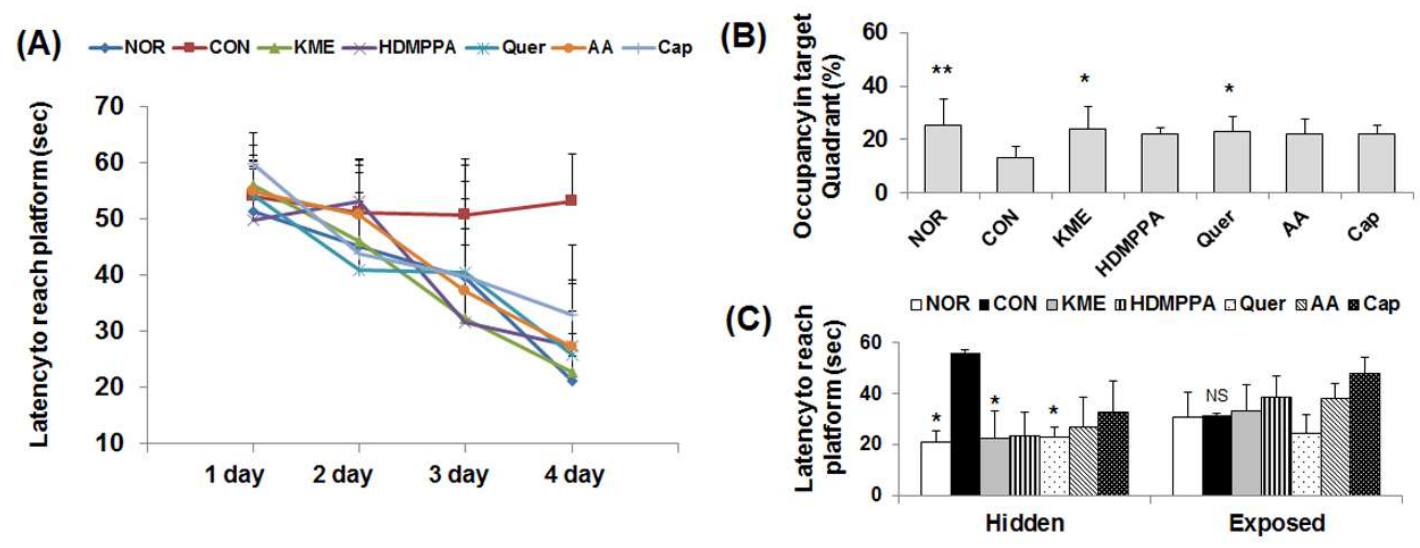

Figure 2. Effects of kimchi methanol extract and kimchi bioactive compounds on mouse behavior in the Morris water maze test. (A) The latency time required to find the platform for 4 days. (B) Occupancy in the target quadrant without the platform on the final test day. (C) The latency time required to reach the hidden and exposed platforms on the final test day. Data are the mean \pm SD ( $n=7$ each group). ${ }^{*} p<0.05,{ }^{* *} p<0.01$ versus the CON group. ${ }^{\text {NS }}$ No significance. (NOR: normal; CON: control; KME: kimchi methanol extract; HDMPPA: 3-(4'-hydroxyl-3',5'-dimethoxyphenyl)propionic acid; Quer: quercetin; AA: ascorbic acid; Cap: capsaicin)

\subsection{Novel Object Recognition Task}

The $\mathrm{A} \beta_{25-35}$-injected $\mathrm{CON}$ group exhibited a poor ability to recognize the new object (Figure 3). Compared with that observed in the $\mathrm{CON}$ group, cognitive function with respect to the frequency of touching the novel object was significantly increased in the KME group, followed by the quercetin, HDMPPA, and ascorbic acid groups, in order $(p<0.05)$. The effect of KME, quercetin, HDMPPA, and ascorbic acid groups were higher by $119.5 \%(p<0.01), 116.9 \%(p<0.01), 114.2 \%(p<0.05)$, and $114.2 \%(p<0.05)$, compared with that of CON group. The CON and capsaicin groups showed no differences in recognition of the different objects.

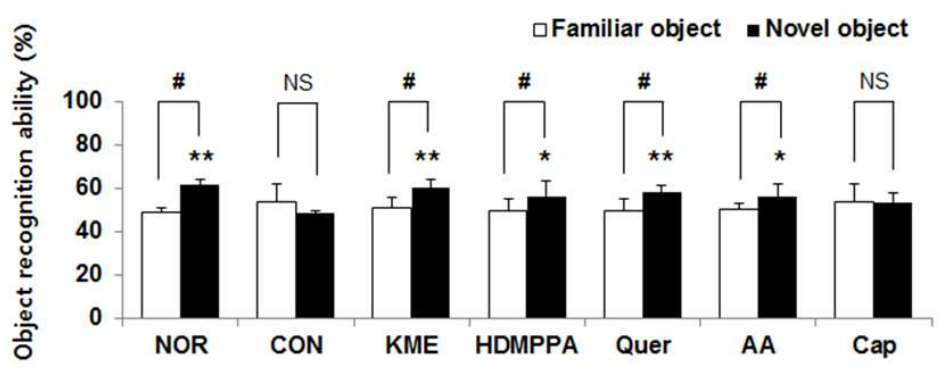

Figure 3. Effects of kimchi methanol extract and kimchi bioactive compounds on mouse behavior in the object recognition test. Data are the mean $\pm \operatorname{SD}\left(n=7\right.$ each group). ${ }^{*} p<0.05,{ }^{* *} p<0.01$ versus the CON group. ${ }^{N S}$ No significance. \# The cognitive abilities for recognizing original and novel objects were significantly different as determined by Student's $t$-test $(p<0.05)$.

\subsection{T-Maze Test}

The KME group demonstrated the highest frequencies of entering a new gate to find the maze, followed by the HDMPPA, quercetin, ascorbic acid, and capsaicin groups. In particular, the KME and quercetin groups showed significantly greater spatial cognition than did the CON group $(p<0.05$, Figure 4). 


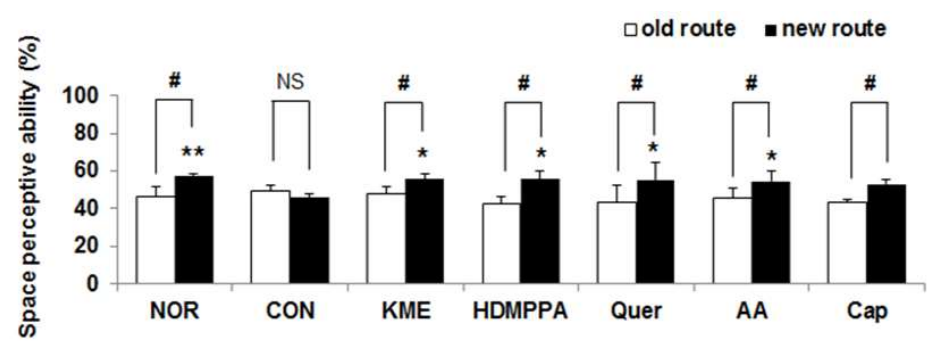

Figure 4. Effects of kimchi methanol extract and kimchi bioactive compounds on mouse behavior in the T-maze test. Data are the mean $\pm \mathrm{SD}$ ( $n=7$ each group). ${ }^{*} p<0.05,{ }^{* *} p<0.01$ versus the CON group. NS No significance. \# The spatial perception abilities for old and new routes were significantly different as determined by Student's $t$-test $(p<0.05)$.

\subsection{Inhibition of Oxidative Stress in the Brain Tissue}

The concentrations of ROS, peroxynitrite, and TBARS in the brain tissue of the CON mice were significantly higher than those of the NOR mice, whereas the GSH level was lower $(p<0.05$, Table 1$)$. Compared with their levels in the CON group, the ROS and peroxynitrite levels in the quercetin group were $42.7 \%$ and $52.3 \%$ lower, respectively $(p<0.05)$. The TBARS level of the KME group was the lowest, followed by that of the quercetin and HDMPPA groups, of which the concentration was decreased by $34.6 \%, 23.0 \%$, and $22.8 \%$, respectively, relative to the CON group level. In contrast, the GSH level was significantly increased in the KME and quercetin groups, by $104.2 \%$ and $103.7 \%$, respectively $(p<0.05)$, relative to the level in the $\mathrm{CON}$ group.

Table 1. Reactive oxygen species, peroxynitrite, thiobarbituric acid reactive substances, and glutathione levels in the brain of $A \beta_{25}-35$-injected ICR mice.

\begin{tabular}{|c|c|c|c|c|}
\hline \multirow{2}{*}{ Group } & ROS & Peroxynitrite & TBARS & GSH \\
\hline & \multicolumn{2}{|c|}{ (Flu/min/mg Tissue) } & \multicolumn{2}{|c|}{ (mM/g Tissue) } \\
\hline NOR & $999 \pm 82 * *$ & $582 \pm 91$ * & $78 \pm 27^{* * *}$ & $24.8 \pm 0.5^{* *}$ \\
\hline $\mathrm{CON}$ & $2143 \pm 336$ & $1338 \pm 103$ & $138 \pm 20$ & $23.2 \pm 0.7$ \\
\hline KME & $1258 \pm 192 *$ & $654 \pm 193 *$ & $90 \pm 16^{* * *}$ & $24.6 \pm 0.2 *$ \\
\hline HDMPPA & $1287 \pm 321 *$ & $693 \pm 228 *$ & $107 \pm 7^{*}$ & $23.9 \pm 0.4$ \\
\hline Quer & $1228 \pm 335$ * & $637 \pm 346^{*}$ & $107 \pm 8^{*}$ & $24.8 \pm 0.8 *$ \\
\hline AA & $1539 \pm 466$ & $648 \pm 157^{*}$ & $116 \pm 14$ & $24.1 \pm 1.2$ \\
\hline Cap & $1650 \pm 604$ & $774 \pm 465$ & $120 \pm 4$ & $23.3 \pm 0.5$ \\
\hline
\end{tabular}

Data are the mean $\pm \operatorname{SD}\left(n=7\right.$ each group). ${ }^{*} p<0.05,{ }^{* *} p<0.01$, and ${ }^{* * *} p<0.005$ versus the CON group. (NOR: normal; CON: control; KME: kimchi methanol extract; HDMPPA: 3-(4'-hydroxyl-3', 5'-dimethoxyphenyl) propionic acid; Quer: quercetin; AA: ascorbic acid; Cap: capsaicin; ROS: reactive oxygen species; TBARS: thiobarbituric acid reactive substances; GSH: glutathione)

\subsection{Elevation of the Antioxidative Status in the Brain Tissue}

Compared with its level in the CON group, the protein expression level of the transcription factor Nrf2 was significantly elevated in the KME, HDMPPA, and quercetin groups, by $213.4 \%(p<0.01)$, $172.8 \%(p<0.05)$, and $181.7 \%(p<0.05)$, respectively (Figure 5). The protein expression level of SOD1 was also significantly higher in the KME, HDMPPA, quercetin, and ascorbic acid groups, by $174.4 \%$, $179.7 \%, 175.1 \%$, and $176.8 \%$, respectively ( $p<0.05$ ). In addition, the protein expression level of GPx was significantly higher in the KME, HDMPPA, and quercetin groups, by $272.3 \%, 262.5 \%$, and $274.9 \%$, respectively $(p<0.05)$. 


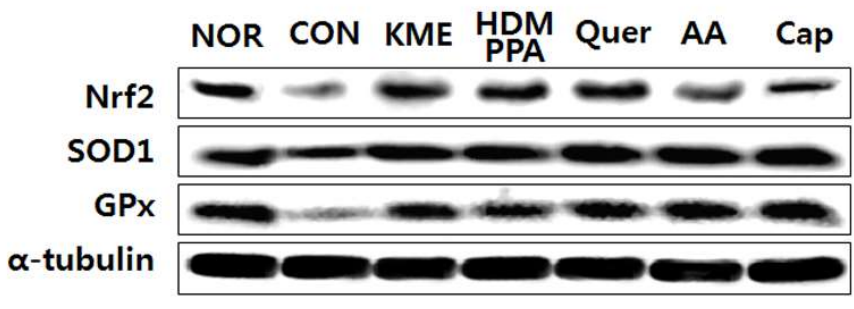

$\square$ NOR $\square$ CON $\square$ KME 目HDMPPA $\square$ Quer $\triangle A A$ Cap

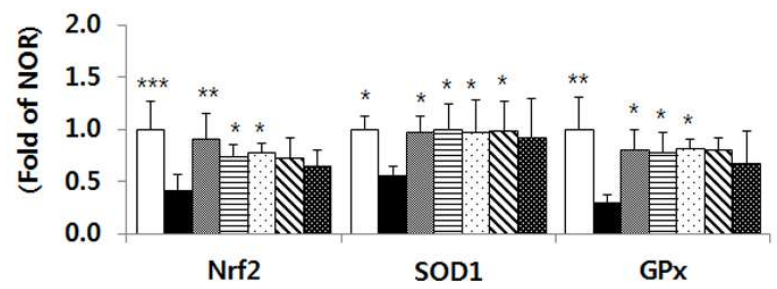

Figure 5. Protein expression levels of antioxidant enzymes in brain tissue of $A \beta_{25-35}$-injected ICR mice. Data are the mean $\pm \mathrm{SD}\left(n=7\right.$ each group). ${ }^{*} p<0.05,{ }^{* *} p<0.01$, and ${ }^{* * *} p<0.005$ versus the CON group.

\subsection{Suppression of the Inflammatory Response in the Brain Tissue}

Compared with its level in the CON group, the protein expression level of the transcription factor NF- $\mathrm{kB}$ was significantly reduced in the KME, HDMPPA, quercetin, and capsaicin groups, by $55.7 \%$ $(p<0.01), 51.6 \%(p<0.01), 52.4 \%(p<0.01)$, and 48.6\% $(p<0.05)$, respectively (Figure 6). The expression level of iNOS was also significantly lower in the KME, HDMPPA, and quercetin groups, by $39.1 \%$ $(p<0.05), 36.1 \%(p<0.05)$, and $45.3 \%(p<0.01)$, respectively. In addition, the protein expression level of COX-2 was significantly lower in the KME, HDMPPA, quercetin, ascorbic acid, and capsaicin groups, by $40.7 \%(p<0.05), 35.3 \%(p<0.01), 35.2 \%(p<0.01), 34.2 \%(p<0.01)$, and $35.6 \%(p<0.01)$, respectively.

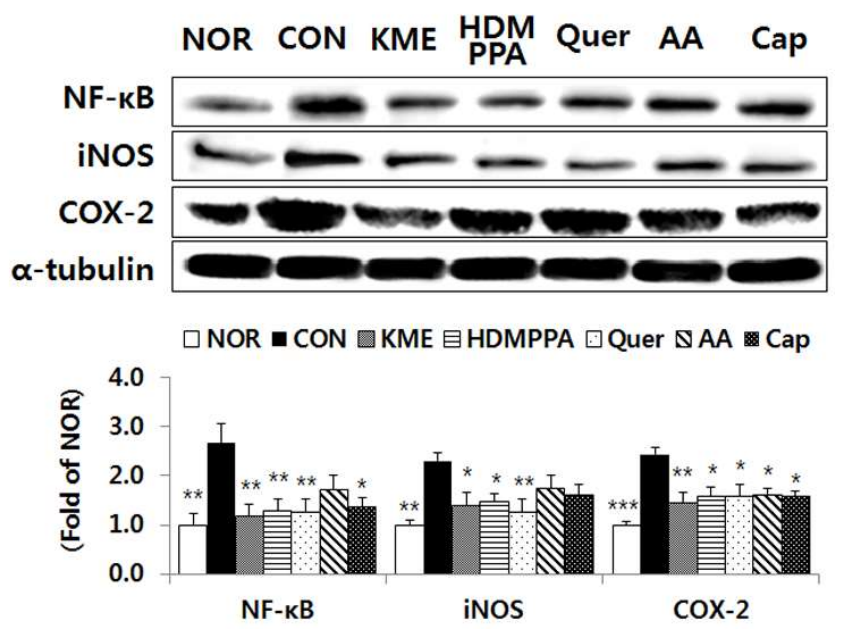

Figure 6. Protein expression levels of inflammation-related molecules in brain tissue of $A \beta_{25-35}$-injected ICR mice. Data are the mean $\pm \mathrm{SD}\left(n=7\right.$ each group). ${ }^{*} p<0.05,{ }^{* *} p<0.01$, and ${ }^{* * *} p<0.005$ versus the CON group.

\section{Discussion}

$\mathrm{AD}$ is the most representative of the age-related progressive neurodegenerative disorders that lead to cognitive deficits $[1,2]$. Behavioral changes are commonly noted in individuals suffering from $\mathrm{AD}$ because cognitive function impairment accompanies a loss of memory or lack of recognition. In the present study, mouse behavior that reflects cognitive function was significantly waned by the injected $A \beta_{25-35}$ peptide. However, the reduced learning and memory abilities were recovered by 
the administered KME and kimchi bioactive compounds, where the latency time to find a hidden platform was decreased, and the abilities to recognize the novel object and new route were promoted. The beneficial effects of KME and kimchi bioactive compounds against AD might be attributed to their antioxidative and anti-inflammatory activities, which inhibit senile plaque formation as well as oxidative stress in the AD-afflicted brain. In the present study, administration of the KME and kimchi bioactive compounds to $A \beta_{25-35}$-injected mice inhibited oxidative stress in the brain. ROS and peroxynitrite generation and TBARS production were reduced with a concomitant increase of the GSH level and Nrf2-regulated antioxidant enzymes. Moreover, we had previously observed that treatment of $\mathrm{A} \beta_{25-35}$-injected mice with KME or kimchi bioactive compounds reduced the levels of amyloid precursor protein (APP), beta-secretase (BACE), and tau proteins in the brain [24]. Blood-brain barrier $(\mathrm{BBB})$ penetration of the natural products is a primary requirement for their ability to protect against degenerative neural diseases. Our team previously examined the BBB penetration ability of some kimchi bioactive compounds and $\mathrm{KME}$, where the in vitro permeability $(\mathrm{Pe})$ values of capsaicin, quercetin, HDMPPA, and KME were 68.3, 14.4, 4.7, and $0.6 \times 10^{-6} \mathrm{~cm} / \mathrm{s}$, respectively, indicating that these bioactive compounds and KME could penetrate the BBB successfully [24]. The permeability of the BBB is controlled by its physicochemical characteristics, and thus hydrophobic and small molecules can readily diffuse into the brain regions [25].

Another critical factor for AD pathology is neuroinflammation injury, the occurrence of which is strongly associated with oxidative stress in the brain. In patients with $A D$, the $A \beta$ peptides induce elevation of the inflammatory cytokines in the brain [26], suggesting that non-steroidal drugs with anti-inflammatory effects could reduce AD risk. In the present study, the KME and kimchi bioactive compounds suppressed the protein expression levels of inflammatory cytokines regulated by NF- $\mathrm{kB}$. In particular, those effects were significantly higher in the KME, HDMPPA, and quercetin groups. Bioactive compounds with antioxidative activity, including phenolic compounds like curcumin and quercetin, have also been shown to mediate neuroprotective effects through the alleviation of oxidative stress [27]. Quercetin diminished the inflammatory response by suppressing NF- $\mathrm{B}$-mediated cytokine generation in vitro [27,28], in human astrocytes [29], and in the brain of d-galactose-treated mice [30]. Similarly, HDMPPA reduced the protein expression level of inflammatory enzymes such as COX-2 and iNOS in mice [31].

The neuroprotective effects of quercetin, HDMPPA, and KME were higher than those of the other bioactive compounds, possibly as a result of their structure specificity. Bioactive compounds that have demonstrated neuronal cell protection against oxidative stress are hydrophobic and have a typical structure, such as a hydroxyl group on the C3 position of the unsaturated C ring [32-34]. Among the bioactive compounds used in this study, quercetin contains a hydroxyl group on the C3 position, and HDMPPA and capsaicin also have a hydroxyl group on the phenol ring. Moreover, quercetin, HDMPPA, and capsaicin are hydrophobic compounds. In addition, all three are phenol-containing compounds, and the antioxidative activity of compounds with phenol moiety is well established [34]. However, the behavioral task test results from our current study do not correlate with the in vitro Pe value. Despite capsaicin having the highest Pe value [24] among the tested compounds, it showed no neuroprotective effects in the AD mice in this study. The possible explanation could be the low dosage (10 mg/kg bw/day) of capsaicin administered to the mice to avoid lethal toxicity. Because the oral $\mathrm{LD}_{50}$ value of capsaicin is $118 \mathrm{mg} / \mathrm{kg}$ bwafter a single dose [19], less than $10 \mathrm{mg} / \mathrm{kg}$ bw/daywas suggested for long-period study [17]. Such a low dose is likely unable to produce neuroprotective effects. In contrast, vitamin C (ascorbic acid) with the lowest $P$ e value of $0.1 \times 10^{-6} \mathrm{~cm} / \mathrm{s}$ among tested compounds demonstrated neuroprotective effects in this study, albeit not as much as those shown by the other bioactive compounds. A previous study reported that the oxidized form of vitamin C (dehydroascorbic acid) could enter the brain and remain in brain tissue in the form of ascorbic acid [33]. Surprisingly, in our present study, the neuroprotective effects of KME in mice were as great as those of quercetin, which showed the greatest effects among the tested kimchi bioactive compounds. This might be due to the high concentration of KME (200 $\mathrm{mg} / \mathrm{kg}$ bw/day) provided, 
on top of the synergic effects from the quercetin, HDMPPA, ascorbic acid, capsaicin, and other possible bioactive compounds in kimchi.

Kimchi has revealed many health benefits [12-14] because its antioxidative potential is high, given that phenolic compounds, carotenoids, flavonoids, vitamin C, and lactic acid bacteria are present in this Korean fermented vegetable [14]. In our previous study, bioactive compounds such as quercetin, capsaicin, ascorbic acid, and HDMPPA were identified in KME [15]. Quercetin, a polyphenolic compound that is present in cabbages or onions, inhibits oxidation by scavenging free radicals [9], suppressing ROS production, and elevating the levels of SOD, GPx, and GSH [32,33]. HDMPPA, an active compound isolated from KME, exhibits antioxidative and anti-inflammatory effects by increasing the GSH concentration and decreasing ROS levels [35]. Capsaicin in red pepper is known to have antioxidative and anticancer properties [36]. In addition, vitamin $\mathrm{C}$ reduces ROS and nitrite production in tissues or plasma [33]. Both kimchi [2] and quercetin [20] were demonstrated to attenuate memory deficits and cognitive impairment in animal models of AD induced by $A \beta_{25-35}$, which are in line with our results. Moreover, several studies have shown that diets rich in antioxidants demonstrate neuroprotective effects against $\operatorname{AD}[9,37,38]$. Curcumin [39], ginkgo biloba [40], and quercetin [9,10,20], all with antioxidative and anti-inflammatory activities, were shown to improve spatial learning and memory abilities by reducing the level of the AD biomarkers APP, BACE, or Tau protein.

In summary, we found that KME and several of its bioactive compounds improved the cognitive functions of mice with $\mathrm{A} \beta_{25-35}$-induced $\mathrm{AD}$, possibly due to their antioxidative and anti-inflammatory activities. Our results could support the agreement among researchers that the amyloid-cascade and the oxidative-stress hypotheses of AD can be considered as one concept [1]. Further studies on the mechanisms of action of the antioxidative and anti-inflammatory compounds capsaicin and ascorbic acid and their association with neuronal diseases are needed. We conclude, however, that the daily consumption of kimchi might be a healthy practice to prevent the onset of AD.

Author Contributions: Y.O.S., and M.J.K. designed the research and provided materials and analysis tools; M.W. performed the experiments, analyzed the data, and wrote the paper; Y.O.S., and M.J.K. reviewed and edited the paper.

Acknowledgments: This research was supported by the Basic Science Research Program through the National Research Foundation of Korea (NRF), funded by the Ministry of Education (2015R1D1A1A01057267).

Conflicts of Interest: The authors declare no conflict of interest.

\section{References}

1. Behl, C.; Moosmann, B. Antioxidant neuroprotection in Alzheimer's disease as preventive and therapeutic approach. Free Radic. Biol. Med. 2002, 33, 182-191. [CrossRef]

2. Lee, A.Y.; Hwang, B.R.; Lee, M.H.; Lee, S.; Cho, E.J. Perilla frutescens var. japonica and rosmarinic acid improve amyloid- $\beta 25-35$ induced impairment of cognition and memory function. Nutr. Res. Pract. 2016, 10, 274-281. [CrossRef] [PubMed]

3. Choi, J.M.; Lee, S.; Park, K.Y.; Kang, S.A.; Cho, E.J. Protective Effect of Kimchi against A $\beta_{25-35}$-induced Impairment of Cognition and Memory. J. Korean Soc. Food Sci. Nutr. 2014, 43, 360-366. [CrossRef]

4. Bevins, R.A.; Besheer, J. Object recognition in rats and mice: A one-trial non-matching-to-sample learning task to study "recognition memory". Nat. Protoc. 2006, 1, 1306-1311. [CrossRef] [PubMed]

5. Montgomery, K.C. A test of two explanations of spontaneous alternation. J. Comp. Physiol. Psychol. 1952, 45, 287-293. [CrossRef] [PubMed]

6. Morris, R. Developments of a water-maze procedure for studying spatial learning in the rat. J. Neurosci. Methods 1984, 11, 47-60. [CrossRef]

7. Gilgun-Sherki, Y.; Melamed, E.; Offen, D. Oxidative stress induced-neurodegenerative diseases: The need for antioxidants that penetrate the blood brain barrier. Neuropharmacology 2001, 40, 959-975. [CrossRef]

8. Essa, M.M.; Vijayan, R.K.; Castellano-Gonzalez, G.; Memon, M.A.; Braidy, N.; Guillemin, G.J. Neuroprotective effect of natural products against Alzheimer's disease. Neurochem. Res. 2012, 37, 1829-1842. [CrossRef] [PubMed] 
9. Wang, L.; Cheng, X.; Li, H.; Qiu, F.; Yang, N.; Wang, B.; Lu, H.; Wu, H.; Shen, Y.; Wang, Y. Quercetin reduces oxidative stress and inhibits activation of c-Jun N-terminal kinase/activator protein-1 signaling in an experimental mouse model of abdominal aortic aneurysm. Mol. Med. Rep. 2014, 9, 435-442. [CrossRef] [PubMed]

10. Chen, J.; Deng, X.; Liu, N.; Li, M.; Liu, B.; Fu, Q.; Qu, R.; Ma, S. Quercetin attenuates tau hyperphosphorylation and improves cognitive disorder via suppression of ER stress in a manner dependent on AMPK pathway. J. Funct. Foods 2016, 22, 463-476. [CrossRef]

11. Kim, B.K.; Choi, J.M.; Kang, S.A.; Park, K.Y.; Cho, E.J. Antioxidative effects of Kimchi under different fermentation stage on radical-induced oxidative stress. Nutr. Res. Pract. 2014, 8, 638-643. [CrossRef] [PubMed]

12. Woo, M.; Kim, M.; Noh, J.S.; Park, C.H.; Song, Y.O. Preventative activity of kimchi on high cholesterol diet-induced hepatic damage through regulation of lipid metabolism in LDL receptor knockout mice. Food Sci. Biotechnol. 2018, 27, 211-218. [CrossRef] [PubMed]

13. Kim, B.; Song, J.-L.; Ju, J.-H.; Kang, S.-A.; Park, K.-Y. Anticancer effects of kimchi fermented for different times and with added ingredients in human HT-29 colon cancer cells. Food Sci. Biotechnol. 2015, 24, 629-633. [CrossRef]

14. Park, K.-Y.; Jeong, J.-K.; Lee, Y.-E.; Daily, J.W., III. Health benefits of kimchi (Korean fermented vegetables) as a probiotic food. J. Med. Food 2014, 17, 6-20. [CrossRef] [PubMed]

15. Woo, M.; Kim, M.; Noh, J.S.; Song, Y.O. Kimchi methanol extracts attenuate hepatic steatosis induced by high cholesterol diet in low-density lipoprotein receptor knockout mice through inhibition of endoplasmic reticulum stress. J. Funct. Foods 2017, 32, 218-225. [CrossRef]

16. Laursen, S.E.; Belknap, J. Intracerebroventricular injections in mice: Some methodological refinements. J. Pharmacol. Methods 1986, 16, 355-357. [CrossRef]

17. Ohnuki, K.; Haramizu, S.; Oki, K.; Watanabe, T.; Yazawa, S.; Fushiki, T. Administration of capsiate, a non-pungent capsaicin analog, promotes energy metabolism and suppresses body fat accumulation in mice. Biosci. Biotechnol. Biochem. 2001, 65, 2735-2740. [CrossRef] [PubMed]

18. Konopacka, M.; Widel, M.; Rzeszowska-Wolny, J. Modifying effect of vitamins C, E and beta-carotene against gamma-ray-induced DNA damage in mouse cells. Mutat. Res. Genet. Toxicol. Environ. Mutagen. 1998, 417, 85-94. [CrossRef]

19. Saito, A.; Yamamoto, M. Acute oral toxicity of capsaicin in mice and rats. J. Toxicol. Sci. 1996, 21, 195-200. [CrossRef] [PubMed]

20. Kim, J.H.; Lee, J.; Lee, S.; Cho, E.J. Quercetin and quercetin-3- $\beta$-d-glucoside improve cognitive and memory function in Alzheimer's disease mouse. Appl. Biol. Chem. 2016, 59, 721-728. [CrossRef]

21. Ali, S.; LeBel, C.; Bondy, S. Reactive oxygen species formation as a biomarker of methylmercury and trimethyltin neurotoxicity. Neurotoxicology 1991, 13, 637-648.

22. Kooy, N.W.; Royall, J.A.; Ischiropoulos, H.; Beckman, J.S. Peroxynitrite-mediated oxidation of dihydrorhodamine 123. Free Radic. Biol. Med. 1994, 16, 149-156. [CrossRef]

23. Jung, K.; Hong, S.H.; Kim, M.; Han, J.-S.; Jang, M.-S.; Song, Y.O. Antiatherogenic effects of Korean cabbage kimchi with added short arm octopus. Food Sci. Biotechnol. 2015, 24, 249-255. [CrossRef]

24. Woo, M.; Noh, J.S.; Cho, E.J.; Song, Y.O. Bioactive Compounds of Kimchi Inhibit Apoptosis by Attenuating Endoplasmic Reticulum Stress in the Brain of Amyloid B-Injected Mice. J. Agric. Food Chem. 2018, 66, 4883-4890. [CrossRef] [PubMed]

25. Youdim, K.A.; Qaiser, M.Z.; Begley, D.J.; Rice-Evans, C.A.; Abbott, N.J. Flavonoid permeability across an in situ model of the blood-brain barrier. Free Radic. Biol. Med. 2004, 36, 592-604. [CrossRef] [PubMed]

26. Akiyama, H.; Barger, S.; Barnum, S.; Bradt, B.; Bauer, J.; Cole, G.M.; Cooper, N.R.; Eikelenboom, P.; Emmerling, M.; Fiebich, B.L. Inflammation and Alzheimer's disease. Neurobiol. Aging 2000, 21, $383-421$. [CrossRef]

27. Banerjee, T.; Van der Vliet, A.; Ziboh, V. Downregulation of COX-2 and iNOS by amentoflavone and quercetin in A549 human lung adenocarcinoma cell line. Prostaglandins Leukot. Essent. Fatty Acids 2002, 66, 485-492. [CrossRef] [PubMed] 
28. García-Mediavilla, V.; Crespo, I.; Collado, P.S.; Esteller, A.; Sánchez-Campos, S.; Tuñón, M.J.; González-Gallego, J. The anti-inflammatory flavones quercetin and kaempferol cause inhibition of inducible nitric oxide synthase, cyclooxygenase- 2 and reactive C-protein, and down-regulation of the nuclear factor kappaB pathway in Chang Liver cells. Eur. J. Pharmacol. 2007, 557, 221-229. [CrossRef] [PubMed]

29. Sharma, V.; Mishra, M.; Ghosh, S.; Tewari, R.; Basu, A.; Seth, P.; Sen, E. Modulation of interleukin-1 $\beta$ mediated inflammatory response in human astrocytes by flavonoids: Implications in neuroprotection. Brain Res. Bull. 2007, 73, 55-63. [CrossRef] [PubMed]

30. Sun, S.W.; Yu, H.Q.; Zhang, H.; Zheng, Y.L.; Wang, J.J.; Luo, L. Quercetin attenuates spontaneous behavior and spatial memory impairment in d-galactose-treated mice by increasing brain antioxidant capacity. Nutr. Res. 2007, 27, 169-175. [CrossRef]

31. Noh, J.S.; Choi, Y.H.; Song, Y.O. Beneficial effects of the active principle component of Korean cabbage kimchi via increasing nitric oxide production and suppressing inflammation in the aorta of apoE knockout mice. Br. J. Nutr. 2013, 109, 17-24. [CrossRef] [PubMed]

32. Ishige, K.; Schubert, D.; Sagara, Y. Flavonoids protect neuronal cells from oxidative stress by three distinct mechanisms. Free Radic. Biol. Med. 2001, 30, 433-446. [CrossRef]

33. Heo, J.-H.; Hyon-Lee; Lee, K.-M. The possible role of antioxidant vitamin C in Alzheimer's disease treatment and prevention. Am. J. Alzheimer's Dis. 2013, 28, 120-125. [CrossRef] [PubMed]

34. Rice-Evans, C.A.; Miller, N.J.; Paganga, G. Structure-antioxidant activity relationships of flavonoids and phenolic acids. Free Radic. Biol. Med. 1996, 20, 933-956. [CrossRef]

35. Noh, J.S.; Kim, H.J.; Kwon, M.J.; Song, Y.O. Active principle of kimchi, 3-(4'-hydroxyl-3' $5^{\prime}$-dimethoxyphenyl) propionic acid, retards fatty streak formation at aortic sinus of apolipoprotein E knockout mice. J. Med. Food 2009, 12, 1206-1212. [CrossRef] [PubMed]

36. Pyun, C.W.; Kim, J.H.; Han, K.H.; Hong, G.E.; Lee, C.H. In vivo protective effects of dietary curcumin and capsaicin against alcohol-induced oxidative stress. BioFactors 2014, 40, 494-500. [CrossRef] [PubMed]

37. Ye, X.; Bhupathiraju, S.N.; Tucker, K.L. Variety in fruit and vegetable intake and cognitive function in middle-aged and older Puerto Rican adults. Br. J. Nutr. 2013, 109, 503-510. [CrossRef] [PubMed]

38. Sabogal-Guáqueta, A.M.; Munoz-Manco, J.I.; Ramírez-Pineda, J.R.; Lamprea-Rodriguez, M.; Osorio, E.; Cardona-Gómez, G.P. The flavonoid quercetin ameliorates Alzheimer's disease pathology and protects cognitive and emotional function in aged triple transgenic Alzheimer's disease model mice. Neuropharmacology 2015, 93, 134-145. [CrossRef] [PubMed]

39. Hamaguchi, T.; Ono, K.; Yamada, M. Curcumin and Alzheimer's disease. CNS Neurosci. Ther. 2010, 16, 285-297. [CrossRef] [PubMed]

40. Zeng, K.; Li, M.; Hu, J.; Mahaman, Y.A.; Bao, J.; Huang, F.; Xia, Y.; Liu, X.; Wang, Q.; Wang, J.-Z. Ginkgo biloba extract EGb761 attenuates Hyperhomocysteinemia-induced AD like tau hyperphosphorylation and cognitive impairment in rats. Curr. Alzheimer Res. 2018, 15, 89-99. [CrossRef] [PubMed] 